\title{
STATISTICAL PROPERTIES OF DYNAMICAL CHAOS
}

\author{
Vadim S. AnishChEnKo \\ Institute of Nonlinear Dynamics, Department of Physics, Saratov State University \\ 83, Astrakhanskaya str., 410012, Saratov, Russia \\ TATJANA E. VAdivasova \\ Institute of Nonlinear Dynamics, Department of Physics, Saratov State University \\ 83, Astrakhanskaya str., 410012, Saratov, Russia \\ Galina I. Strelkova \\ Institute of Nonlinear Dynamics, Department of Physics, Saratov State University \\ 83, Astrakhanskaya str., 410012, Saratov, Russia

\section{George A. Okrokvertskhov} \\ Institute of Nonlinear Dynamics, Department of Physics, Saratov State University \\ 83, Astrakhanskaya str., 410012, Saratov, Russia
}

(Communicated by Stefano Boccaletti)

\begin{abstract}
This study presents a survey of the results obtained by the authors on statistical description of dynamical chaos and the effect of noise on dynamical regimes. We deal with nearly hyperbolic and nonhyperbolic chaotic attractors and discuss methods of diagnosing the type of an attractor. We consider regularities of the relaxation to an invariant probability measure for different types of attractors. We explore peculiarities of autocorrelation decay and of power spectrum shape and their interconnection with Lyapunov exponents, instantaneous phase diffusion and the intensity of external noise. Numeric results are compared with experimental data.
\end{abstract}

1. Introduction. Dynamical chaos, like a random process, requires a statistical description. When chaotic systems are studied in computer or physical experiments, probability characteristics (such as a stationary probability distribution on an attractor, correlation functions, power spectra and others) are usually calculated or measured. Chaotic oscillations that correspond to different types of chaotic attractors in the phase space of dynamical systems are characterized by various statistical properties as well as by a different degree of sensitivity of the statistical characteristics to the influence of noise.

In the rigorous theory, hyperbolic chaos is often called "true" chaos and is characterized by a homogeneous and topologically stable structure $[1,2,3,4]$. However, strange chaotic attractors of dynamical systems are not, as a rule, robust hyperbolic sets. Rather they are referred to as nearly hyperbolic attractors; for example, the Lorenz attractor. Nearly hyperbolic (quasihyperbolic) attractors include some

2000 Mathematics Subject Classification. 92D30.

Key words and phrases. nonhyperbolic attractors, spiral and funnel chaos, autocorrelation function, instantaneous phase, phase variance, effective diffusion coefficient. 
nonrobust orbits (for example, separatrix loops), but their appearances and disappearances often do not affect the observed characteristics of chaos, such as a phase portrait, the power spectrum, Lyapunov exponents, and others. Dynamical systems in a chaotic regime may give rise to an invariant measure that does not depend on an initial distribution and fully reflects the statistical properties of the attractor. The existence of an invariant measure has been theoretically proven for hyperbolic and nearly hyperbolic systems $[5,6,7,8,9,10]$.

However, most chaotic attractors that we deal with in numeric simulation and real experiments are nonhyperbolic $[11,12,13]$. The problem of the existence of an invariant measure on a nonhyperbolic chaotic attractor involves serious difficulties because it is generally impossible to obtain a stationary probability distribution that is independent of an initial distribution. A nonhyperbolic attractor is a maximal attractor of the dynamical system and encloses a countable set of regular and chaotic attracting subsets $[11,12]$. When $\delta$-correlated Gaussian noise is added to the system, an invariant measure on such attractors exists too [14]. In the nonhyperbolic case the behavior of phase trajectories is significantly affected by noise $[15,16,17,18]$ while it changes only slightly in systems with hyperbolic and nearly hyperbolic chaos $[15,16,19,20]$.

A statistical description of noisy nonhyperbolic chaotic attractors is an important and still unsolved problem of the dynamical systems theory. One of the topical problems in this direction is to study the relaxation to stationary distributions in time. A number of fundamental questions still have unclear answers. What is a real relaxation time of the system to a stationary distribution? Which factors define this time? Which characteristics can quantify the relaxation time to the stationary measure? What is the role of the noise statistics and the noise intensity in regularities of the relaxation to the stationary distribution? Is there any connection between the relaxation process and the system dynamics? These problems are studied in $[21,22]$ with the methods of computer simulation.

The relaxation to a stationary distribution is described by the evolutionary operator that can be represented by the Fokker-Planck operator or the FrobeniusPerron operator. The eigenvalues and eigenfunctions of the evolutionary operator determine the rate and character of the relaxation process and characteristics of mixing, which are related to the relaxation to an invariant probability measure. However, if the dynamical system is high-dimensional $(N \geq 3)$, the nonstationary solution of the Fokker-Planck equation is difficult to find even numerically. Therefore, the method of stochastic differential equations was used in the studies described in $[21,22]$.

As is commonly known, chaotic dynamics means the presence of mixing and, consequently, is characterized by the positive Kolmogorov entropy. The presence of mixing causes autocorrelation functions (ACF) to decay to zero for large times (correlation splitting). This implies that the system states separated by a sufficiently large time interval become statistically independent [6, 8, 23, 24, 25]. From the property of mixing it follows that a dynamical system is ergodic. For chaotic dynamical systems the splitting of correlations in time is connected with an instability of chaotic trajectories and with the system property to produce entropy $[6,8,23,24,25,26,27]$. In spite of their significant importance, correlation properties of chaotic processes have been studied insufficiently. It is widely believed that autocorrelation functions of chaotic systems exponentially decrease at a rate being defined by the Kolmogorov entropy [23]. The Kolmogorov entropy, $H_{\mathrm{K}}$, in turn is 
bounded from above by the sum of positive Lyapunov exponents [8, 27, 28]. But this estimation is true only for some special cases.

It has been proven for some classes of discrete maps (expanding and Anosov ones), which exhibit a mixing invariant measure, that the decay of correlations with time is bounded from above by an exponential function [9, 29, 30, 31]. There are different estimations of the rate of this exponential decay, which are not always connected with Lyapunov exponents [21, 32, 33, 34]. For continuous-time systems, there are no theoretical results at all for estimating the rate of correlation splitting [35].

The studies of specific chaotic systems testify to a complicated behavior of correlation functions, which is defined not only by positive Lyapunov exponents but also by different characteristics and peculiarities of the system chaotic dynamics $[21,32,34,36]$.

In $[37,38,39]$, the correlation and spectral properties of chaotic oscillations are studied for several types of chaotic attractors that can be observed in autonomous differential systems with three-dimensional phase space. Classical models of nonlinear dynamics, such as the Rössler oscillator [40], the Lorenz system [41], and the Anishchenko-Astakhov oscillator that represents a mathematical model of a real radiotechnical device $[42,43]$, were chosen for the studies. In the cited papers an attempt was taken to answer several fundamental questions. Which peculiarities of the system's chaotic dynamics can define the rate of correlation decay and the basic spectral line width? How does noise affect the spectral and correlation characteristics of chaos? Using the results of numerical simulation, we would like to show that in the context of correlation properties, different types of chaotic selfsustained oscillations can be associated with basic models of stochastic processes, such as harmonic noise and a telegraph signal.

The aim of this work is to present a brief review of the recent results reported in $[21,22,37,38,39]$. The presented results concern the method of diagnosing nonhyperbolic chaos, the influence of noise on nonhyperbolic attractors, some probabilistic aspects of chaotic dynamics (such as peculiarities of the relaxation to a stationary probability distiribution), the rate of mixing, and the correlation and spectral analysis of chaotic regimes of different types. Special attention is paid to the effect of noise on the statistical properties of chaotic dynamics.

2. Diagnostics of hyperbolicity in chaotic systems. Strange attractors in finite-dimensional systems can be divided into three main classes: robust hyperbolic, almost hyperbolic (quasi-hyperbolic) and nonhyperbolic [11, 12, 13]. The property of robust hyperbolicity of a chaotic attractor means that all its trajectories are of the same saddle type, and their stable and unstable manifolds are transversal everywhere; that is, the structure of a hyperbolic attractor is homogeneous in every point on the attractor. In addition, these properties are preserved under small perturbations of the system's parameters. However, robust hyperbolic attractors, such as Smale-Williams' solenoid [44] or Plykin's attractor [45], are almost ideal objects. The existence of robust hyperbolic attractor has been proven for none of the dynamical systems defined in the form of differential equations or discrete maps. Nevertheless, there are several examples of almost hyperbolic attractors, including the Lorenz attractor [46] and the Shimizu-Morioka attractor [47] in flow systems, and the Lozi attractor [48] and the Belykh attractor [49] in discrete maps. For these systems, the presence of singular phase trajectories is typical; for example, 
the Lorenz attractor is characterized by the presence of a set of separatrix loops of a saddle-type equilibrium point in its parameter space; the Lozi attractor encloses nonrobust homoclinic curves without tangencies between some stable and unstable manifolds. These singular trajectories do not lead to the birth of stable motions. Given the behavior of experimentally observed characteristics, quasi-hyperbolic attractors are quite similar to hyperbolic ones.

The majority of chaotic attractors of dynamical systems is, however, nonhyperbolic [11, 12, 13]. Nonhyperbolic attractors include chaotic limit sets as well as stable periodic orbits. The latters are often difficult to detect in numerical experiments because the size of their basins of attraction often appears to be below the double precision of the computer resolution. Nevertheless, even in the presence of noise, the properties of nonhyperbolic attractors are essentially different from those of hyperbolic attractors $[13,50,51]$. The diagnosis of the attractor type is, therefore, very important from theoretical and practical viewpoints; it has strong consequences for the analysis of nonlinear systems, especially for modeling or shadowing $[17,52,53,54,55,56,57]$.

A direct method for detecting if a chaotic system is hyperbolic, is to calculate the angles $\phi$ between the stable and unstable manifolds along a trajectory in points $x$ of the chaotic attractor. A numerical procedure for calculating these $\phi$ was proposed in [58] for diagnosing the hyperbolicity of chaotic saddles in 2-dimensional systems. This method entails in the transformation of an arbitrary vector by the linearized evolution operator along a trajectory forward and backward with allows one to find the angle between the directions of stability and unstability for different points on the chaotic sets.

Manifolds in two-dimensional systems are one-dimensional and the diagnostics of homoclinic tangency has no principal difficulties. For three-dimensional systems the procedure becomes more complicated because in this case manifolds are twodimensional. In [59], we proposed a method for diagnosing the hyperbolicity for three-dimensional differential systems. It was experimentally established that such systems as the Rössler system, Chua's circuit [60], and the Anishchenko-Astakhov oscillator are typically nonhyperbolic ones; that is, they are structurally unstable [11]. The Lorenz system can be treated as an exceptional case. In a certain parameter range the Lorenz attractor is nearly hyperbolic. Stable and unstable manifolds of the attractor trajectory intersect transversally. This property is preserved in the presence of small external noise [59]. However, the Lorenz attractor demonstrates a bifurcational transition into the nonhyperbolicity mode [61]. This effect can be diagnosed by calculating the angles between the manifolds. Figure 1a shows the angle probability distribution $p(\phi)$ for the classical Lorenz attractor that is realized in the following system [41]:

$$
\dot{x}=-\sigma(x-y), \dot{y}=r x-y-x z, \dot{z}=-\beta z+x y .
$$

As seen from the first plot, the probability of homoclinic tangency is rigorously equal to zero $(p(\phi)=0)$. The escape from the region where the Lorenz attractor exists leads to the appearance of the effect of homoclinic tangency (Fig. 1b). In this case, we observe that for $r \geq 38$ the angle between the manifolds becomes equal to zero (Fig. 1b). This effect mainly explains the properties and peculiarities of chaos, which are discussed in further sections of this paper. Our numeric results have also shown that the presence of small additive noise sources does not significantly affect the structure of angle probability distributions for either nearly hyperbolic 
(a)

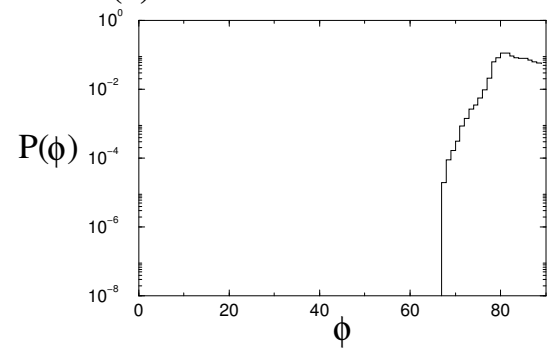

(b)

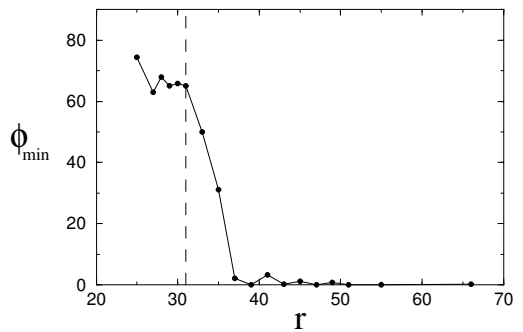

Figure 1. Calculation results for the Lorenz system (1) for $\sigma=10$ and $b=8 / 3$. (a) The angle probability distribution for the Lorenz attractor with $r=27$; (b) the dependence of minimal angle $\phi_{\min }$ on parameter $r$. The dashed line denotes the theoretical onset of the trasition from the Lorenz attractor to nonhyperbolic attractors.

and nonhyperbolic attractors. The character of a chaotic set remains the same under influence of noise [59].

3. Chaos in the presence of noise. Stochastic nonlinear problems are of fundamental and practical importance. They can be treated as a natural extension of the nonlinear dynamics problems. The presence of noise in a system requires a transition to a statistical description. There are two main approaches to studying stochastic systems $[62,63,64,65]$. The first one is based on solving stochastic equations (SE) and is also called Langevin's method. Each particular solution of $\mathrm{SE}$, even with the same initial state, produces a new realization of a random process. By this means, one is able to obtain a statistical ensemble of a great number of realizations and to find its intrinsic statistical characteristics. In practice, time averaging along a single, long-enough realization is often used, assuming that the process is ergodic. The second approach consists of solving evolution equations called the Chapman-Kolmogorov equation, the kinetic equation, or the FokkerPlanck equation - for the probability measure. However, the process in a system should be at least Markovian, which imposes certain requirements on the noise sources. For theprocess to be Markovian, random kicks must be statistically independent. In this case the Chapman-Kolmogorov equation holds. If the noise possesses Gaussian properties, the process is diffusive; therefore, for the probability density we can write the Fokker-Planck equation. Under appropriate definitions of noise sources, the method of SE and the method of evolution equations must yield the equivalent statistical description of the system $[62,63,64,66]$.

Of special interest is the problem of statistical characteristics of dynamical chaos and of the role of fluctuations in chaotic systems [6, 7, 19, 42, 66, 67, 68, 69, 70]. For systems with chaotic dynamics of the hyperbolic type, the transition to a statistical description already is possible in a purely deterministic case; that is, without noise $[6,7,19,70]$. This means that the stationary solution of the evolution equation for the probability density allows the presence of the limit $D \rightarrow 0$, where $D$ is the noise intensity, and there is the possibility of deducing an expression for the probability measure in a purely deterministic case. As shown in $[6,19]$, small fluctuations $(D \ll 1)$ in hyperbolic systems cause small changes of the structure of 
the probability measure. So-called quasi-hyperbolic (almost hyperbolic) attractors, such as the Lozi attractor and the Lorenz attractor [11, 12], behave in a similar manner. Almost hyperbolic attractors enclose non-robust singular trajectories, for example, separatrix loops in the Lorenz attractor. Nevertheless, these attractors, as well as hyperbolic ones, do not contain stable periodic orbits. The characteristics of quasi-hyperbolic attractors, measured in numerical experiments, are robust relative to small perturbations of the evolution operator. Particularly, there is a rigorous proof for the existence of the probability measure of the Lorenz attractor without noise [7]. A system's own dynamics proves to be much stronger than that imposed from outside by external noise [6].

In nonhyperbolic systems the effect of noise can play a significant role. In [16] it was shown that the mean distance between a noisy orbit and the noiseless nonhyperbolic attractor appears to be significantly larger than that in the hyperbolic case and depends on the information dimension of the attractor. It is well known that in systems with nonhyperbolic attractors noise can induce various phase transitions $[42,43,71,72]$. When Gaussian noise sources are added to a system, basins of attraction of all the system's attractors can merge. As a result, a unified stationary probability density appears, independent of the initial state [14]. However, the statistical description of nonhyperbolic chaos encounters principal difficulties. In general, there is no stationary probability measure on nonhyperbolic chaotic attractors without noise, independent of the initial distribution. In this case the continuous limit transition $D \rightarrow 0$ does not exist for the probability density of noisy nonhyperbolic systems [14]. Moreover, the probability characteristics of nonhyperbolic chaos are very sensitive to even the slightest changes of the system parameters $[13,42,59,73]$.

\section{Relaxation to a stationary probability distribution of chaotic attrac- tors in the presence of noise.}

4.1. Models and numerical methods. Let us consider chaotic attactors of wellknown model systems, such as the Rössler oscillator [40]

$$
\dot{x}=-y-z+\sqrt{2 D} \xi(t), \dot{y}=x+a y, \dot{z}=b-z(m-x),
$$

and the Lorenz system (1) [41] with noise

$$
\dot{x}=-\sigma(x-y)+\sqrt{2 D} \xi(t), \dot{y}=r x-y-x z, \dot{z}=-\beta z+x y .
$$

In both models, $\xi(t)$ is a Gaussian white noise source with the mean value $\langle\xi(t)\rangle \equiv 0$ and correlation $\langle\xi(t) \xi(t+\tau)\rangle \equiv \delta(\tau)$, where $\delta()$ is Dirac's function. The parameter $D$ denotes the noise intensity. For the Rössler system, we fix $a=0.2$ and $b=0.2$ and vary the control parameter $m$ in the interval $[4.25,13]$. In the Lorenz system, we choose two different regimes, namely, a quasi-hyperbolic attractor $(\sigma=10, \beta=8 / 3$, and $r=28)$ and a nonhyperbolic attractor $(\sigma=10, \beta=8 / 3$, and $r=210)$.

Chaotic attractors of systems (2) and (3) have been studied in detail and are typical examples of quasi-hyperbolic and nonhyperbolic chaos [43, 74]. Thus, results obtained for Equations (2) and (3) can be generalized to a wide class of dynamical systems.

To examine the relaxation to a stationary distribution in these systems, we analyze how points situated at an initial time in a cube of small size $\delta$ around an arbitrary point of the trajectory belonging to an attractor of the system evolve with time. We take $\delta=0.09$ for the size of this cube and fill it uniformly with $n=9000$ 
points. As time goes on, these points in the phase space are distributed throughout the whole attractor. To characterize the convergence to the stationary distribution we follow the temporal evolution of this set of points and calculate the ensemble average

$$
\bar{x}(t)=\int_{W} p(x, t) x d x=\frac{1}{n} \sum_{i=1}^{n} x_{i}(t) .
$$

Here, $x$ is one of the system dynamical variables, and $p(x, t)$ is the probability density of the variable $x$ at the time $t$ which corresponds to the chosen initial distribution. It is known that the phase trajectory of system (3) visits neighborhoods of two saddle-foci. In this case, when calculating $\bar{x}(t)$ one may first sum separately over points having fallen in the neighborhood of each saddle-focus, and then combine the obtained results. However, the mean value appears to approach zero in a short time interval, and its further evolution is badly detected. To follow the relaxation in system (3), we compute the mean value when points in the neighborhood of only one saddle-focus are taken into account. In this case the relaxation to this quantity goes more slowly in time. Then we calculate the function $\gamma\left(t_{k}\right)$ :

$$
\gamma\left(t_{k}\right)=\left|\bar{x}_{m}\left(t_{k+1}\right)-\bar{x}_{m}\left(t_{k}\right)\right|,
$$

where $\bar{x}_{m}\left(t_{k}\right)$ and $\bar{x}_{m}\left(t_{k+1}\right)$ are successive extrema of $\bar{x}(t)$. Thus, $\gamma\left(t_{k}\right)$ characterizes the amplitude of the mean value oscillations. In expression (5), $t_{k}$ and $t_{k+1}$ are successive time moments corresponding to the extrema of $\bar{x}$. The temporal behavior of $\gamma\left(t_{k}\right)$ allows us to judge the character and the rate of relaxation to the probability measure on the attractor. We also calculate the maximal Lyapunov exponent (LE) $\lambda_{1}$ of a chaotic trajectory on an attractor. We also compute the normalized autocorrelation function $(\mathrm{ACF})$ of steady-state oscillations $x(t)$ :

$$
\Psi(\tau)=\psi(\tau) / \psi(0), \quad \psi(\tau)=\langle x(t) x(t+\tau)\rangle-\langle x(t)\rangle\langle x(t+\tau)\rangle .
$$

The brackets $\langle\ldots\rangle$ denote time averaging.

To make some figures more informative and compact, instead of $\gamma\left(t_{k}\right)$ and $\Psi(\tau)$, we plot (where it is necessary) their envelopes $\gamma_{0}\left(t_{k}\right)$ and $\Psi_{0}(\tau)$, respectively.

4.2. Relaxation to a stationary distribution in the Rössler system: Mechanism of the effect of noise on the rate of mixing. A chaotic attractor realizing in the Rössler system (2) at fixed $a=b=0.2$ and in the parameter $m$ interval $[4.25,8.5]$ serves as a well-known example of a spiral attractor. The phase trajectory on the spiral attractor rotates with a high regularity around one or several saddle-foci. The autocorrelation function is oscillating and the power spectrum exhibits narrow-band peaks corresponding to the mean rotation frequency, its harmonics and subharmonics. By virtue of these properties, spiral chaos is called phase-coherent $[43,75,76,77]$.

The chaotic attractor of system (2) is qualitatively changing as the parameter $m$ increases. In the interval $8.5<m<13$, a nonhyperbolic attractor of the noncoherent type, called a funnel attractor occurs $[42,76]$. Phase trajectories on the funnel attractor make complicated loops around a saddle-focus and thus, demonstrate a non-regular rotation behavior. Consequently, the autocorrelation function of noncoherent chaos decreases much more rapidly than that in the coherent case, and the power spectrum does not already contain sharp peaks.

The calculations performed for $m \in[4.25,7.5]$ (spiral chaos) and for $m \in[8.5,13]$ (noncoherent chaos) allow us to assume that an invariant probability measure exists for the parameter values considered. All the effects being observed for each type of 
attractor in system (2) are qualitatively preserved when the parameter $m$ is varied. In our numeric simulation, we fix $m=6.1$ for the spiral attractor and $m=13$ for the funnel attractor.

Figure 2 shows the typical behavior of $\gamma_{0}(t)$ for the spiral and the funnel attractor of the Rössler system. We find that the noise significantly influences the rate of mixing in the regime of spiral attractor in the Rössler system. The relaxation time is strongly decreasing for increasing noise intensity (see Fig. 2a). We find a quite different situation for the funnel attractor. Noncoherent chaos is practically insensitive to noise perturbations. Behavior of $\gamma_{0}\left(t_{k}\right)$ does not significantly change when noise is added to Equations (2) (see Fig. 2b). At the same time, it is well known that noncoherent chaos exhibits a close similarity to random processes. This fact can be verified; for example, by means of the autocorrelation function $\Psi(\tau)$ for the spiral and the funnel attractors in system (2) (Fig. 3). Our numerical experiments show that the correlation times are essentially different for these two chaotic regimes: without noise they differ by two orders. On the one hand, in the case of coherent chaos the correlation time decreases dramatically in the presence of noise (Fig. 3a). On the other hand, the autocorrelation function for the funnel attractor in the deterministic case practically coincides with that in the presence of noise (Fig. 3b). Hence, noncoherent chaos, which is nonhyperbolic, demonstrates some property of hyperbolic chaos, i.e. "dynamical stochasticity" turns out to be much stronger than that imposed from an external (additive) one [6]. This experimental result is interesting and requires a more detailed consideration.

It is also worth noting another finding of our simulations. We have found that the positive LE for the spiral chaos and the funnel chaos is weakly sensitive to fluctuations (see Fig. 4), and decreases somewhat with increasing noise intensity, although in certain cases the correlation time changes considerably under the influence of noise. Thus, in the regime of spiral chaos, the rate of mixing is not uniquely determined by the largest LE but depends strongly on the noise intensity.

We suppose that the essential effect of noise on relaxation to the stationary distribution in the regime of spiral chaos may be associated with peculiarities of the phase trajectory rotation around a saddle-focus. Since the trajectory rotates almost regularly on the spiral attractor, the relaxation process appears to be very
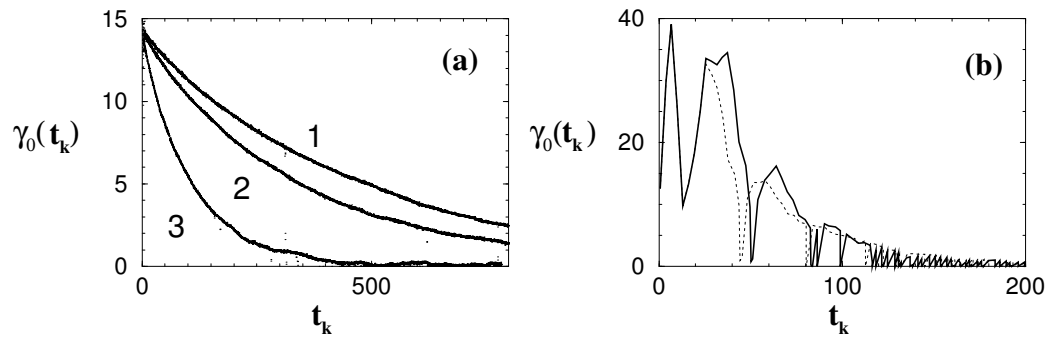

FigURE 2. Behavior of $\gamma_{0}\left(t_{k}\right)$ for attractors in the Rössler system (2). (a) For the spiral attractor $(a=b=0.2, m=6.1)$ for $D=0$ (curve 1), $D=0.001$ (curve 2), and $D=0.1$ (curve 3); (b) For the funnel attractor $(a=b=0.2, m=13)$ for $D=0$ (solid line) and for $D=0.01$ (dotted line). 

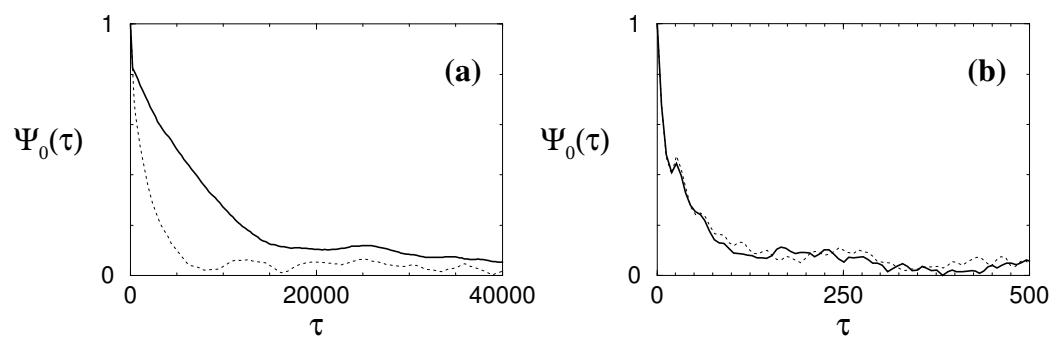

FiguRE 3. Envelopes of the normalized autocorrelation function $\Psi_{0}(\tau)$ for attractors in (2). (a) At $m=6.1$ and for $D=0$ (solid line) and $D=0.01$ (dotted line); (b) At $m=13$ for $D=0$ (solid line) and $D=0.01$ (dotted line).

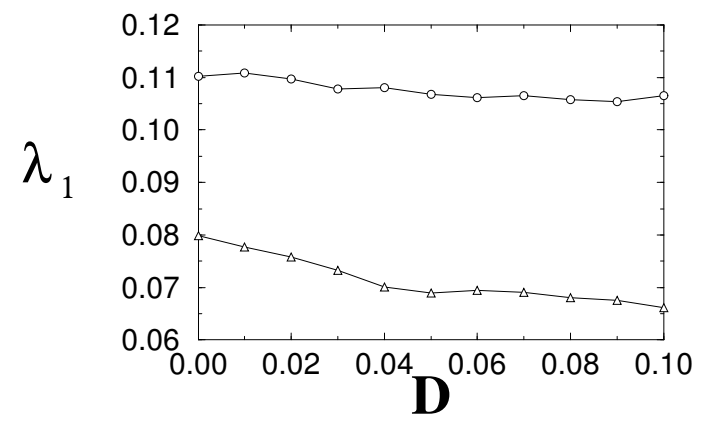

FiguRE 4. For the Rössler system, $\lambda_{1}$ on the spiral (triangles) and the funnel (circles) attractor as functions of the noise intensity $D$.

long. The addition of noise to the system destroys the relative regularity of the trajectory and, consequently, the rate of mixing significantly increases.

It is known that for chaotic oscillations one can introduce the notion of instantaneous amplitude and phase [77]. The instantaneous phase characterizes the rotation of a trajectory around a saddle-focus. System (2) is of such type because the trajectory in the $(x, y)$ projection rotates around the unique saddle-focus located very near to the origin. Let us introduce the substitution of variables

$$
x(t)=A(t) \cos \Phi(t), \quad y(t)=A(t) \sin \Phi(t),
$$

which defines the amplitude $A(t)$ and the total phase $\Phi(t)$ of the chaotic oscillations. Then the instantaneous phase $\Phi(t)$ can be calculated as follows:

$$
\Phi(t)=\arctan \frac{y(t)}{x(t)}+\pi n(t),
$$

where $n(t)=0,1,2, \ldots$ is the number of intersections of the phase trajectory with the plane $x=0$.

The component of mixing along the flow of trajectories is related to the divergence of the instantaneous phase values and thus, is determined by the temporal behavior of the phases. The instantaneous phases of an ensemble of initially close 
trajectories on the spiral attractor remain very close to each other over a long period of time, although the points in the secant plane are spread over the whole attractor section. In this case the relaxation to a stationary probability distribution on the whole attractor of a flow system will be much longer than that in the Poincaré map. The violation of regular rotation of trajectories is characteristic for the funnel attractor and leads to a nonmonotonic dependence of the intantaneous phase on time. The phase trajectory creates complicated loops at nonequal time intervals that causes the value of the current phase to slightly decrease. This results in a rapid divergence of the phase values of neighboring trajectories. The influence of noise on spiral chaos leads to similar effects. Figure 5a shows the temporal dependences of the variance $\sigma_{\Phi}^{2}$ of the instantaneous phase on an ensemble of initially close trajectories for the spiral and the funnel attractor of system (2). We observe that in the noisy and the noise-free cases the variance grows almost linearly on the time intervals being considered. The fact that the temporal dependence of the instantaneous phase variance of the chaotic oscillations in the Rössler system is a linear function was assumed in [75]. Nevertheless, this suggestion was not confirmed theoretically, numerically, or experimentally. In the case of spiral chaos without noise (curve 1), the value of $\sigma_{\Phi}^{2}$ is small (on the given time interval it does not exceed the variation of the uniform phase distribution on the interval $[-\pi ; \pi]$ ) and increases much slower than in the other cases considered. The linear growth of the variance allows us to estimate the divergence of the intantaneous phases by using the effective diffusion coefficient:

$$
B_{\mathrm{eff}}=\left\langle\frac{1}{2} \frac{d \sigma_{\Phi}^{2}(t)}{d t}\right\rangle
$$

where the angle brackets denote time averaging.

Figure $5 \mathrm{~b}$ illustrates the dependences of $B_{\text {eff }}$ of the instantaneous phase of chaotic oscillations on the noise intensity for the spiral and the funnel attractor in the Rössler system (2). In both cases, $B_{\text {eff }}$ grows with increasing $D$, but for spiral chaos this growth is more significant. This result strongly testifies that $B_{\text {eff }}$ is a very effective characteristic for diagnosing the statistical properties of a chaotic attractor.
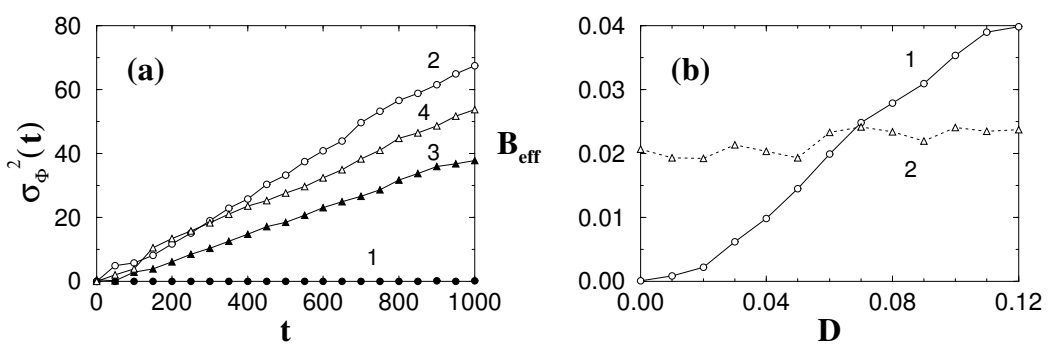

Figure 5. (a) Temporal dependences of the variance of the intantaneous phase $\sigma_{\Phi}^{2}$ for spiral chaos $[m=6.1$ at $D=0$ (curve 1 ), $D=0.1$ (curve 2)]; and for noncoherent chaos $[m=13$ at $D=0$ (curve 3), $D=0.1$ (curve 4]; (b) The effective diffusion coefficient $B_{\text {eff }}$ as a function of the noise intensity $D$ for spiral (curve 1) and noncoherent (curve 2) chaos. 
4.3. Relaxation to a probability measure in the Lorenz system. Wellknown quasi-hyperbolic attractors in three-dimensional continuous-time systems, such as the Lorenz attractor and the Shimizu-Morioka attractor [47], are attractors of the switching type. The phase trajectory switches chaotically from the neighborhood of one saddle equilibrium state to the neighborhood of another one. Such switchings are accompanied by chaotic phase changes even without noise. In this case the addition of noise does not change considerably the phase dynamics and, consequently, does not influence the rate of relaxation to the stationary distribution.

Figure 6 shows the behavior of $\gamma_{0}\left(t_{k}\right)$ for both quasi-hyperbolic and nonhyperbolic chaotic attractors of system (3) with and without noise added. We find that for the Lorenz attractor noise does not significantly influence the relaxation rate (Fig. 6a). However, we observe a quite different situation for the nonhyperbolic attractor in the Lorenz system: The rate of relaxation is strongly affected by noise (Fig. 6b).
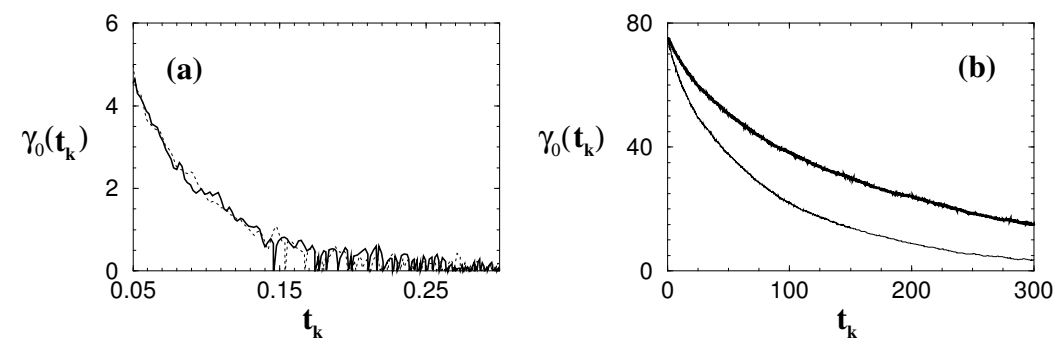

FiguRE 6. Behavior of $\gamma_{0}\left(t_{k}\right)$ for chaotic attractors in the Lorenz system (3). (a) For $r=28$ and $D=0$ (solid line) and for $D=0.01$ (dotted line); (b) For $r=210$ and $D=0$ (thick line), and for $r=210$ and $D=0.01$ (thin line). Other parameters are $\sigma=10$, $\beta=8 / 3$.

Next, we will check whether the other characteristics of the mixing rate, such as the LE and the correlation time, will also depend on noise perturbations. For the same chaotic attractors in the Lorenz system we compute the largest LE $\lambda_{1}$ and estimate the normalized autocorrelation function $\Psi(\tau), \tau=t_{2}-t_{1}$, of the dynamical variable $x(t)$ for different noise intensities $D$. We find that for both types of chaotic attractors the LE does not depend within the calculation accuracy on the noise intensity. The autocorrelation function of the quasi-hyperbolic attractor is essentially unaffected by noise (Fig. 7a). However, in the regime of a nonhyperbolic attractor it decreases more rapidly in the presence of noise (see curves in Fig. 7b).

5. Correlation and spectral analysis of dynamical chaos. Let us now examine correlation and spectral properties of different types of chaotic oscillations in more details. Experience of the studies of dynamical chaos in three-dimensional differential systems shows that two classical models of random processes can be used to describe the correlation and spectral properties of a certain class of chaotic systems. They are the models of harmonic noise and a telegraph signal. As we will demonstrate below, the model of harmonic noise represents sufficiently well correlation characteristics of spiral chaos, while the model of telegraph signal is quite suitable for studying statistical properties of attractors of the switching type, such as attractors in the Lorenz system [41] and in the Chua circuit [60]. 

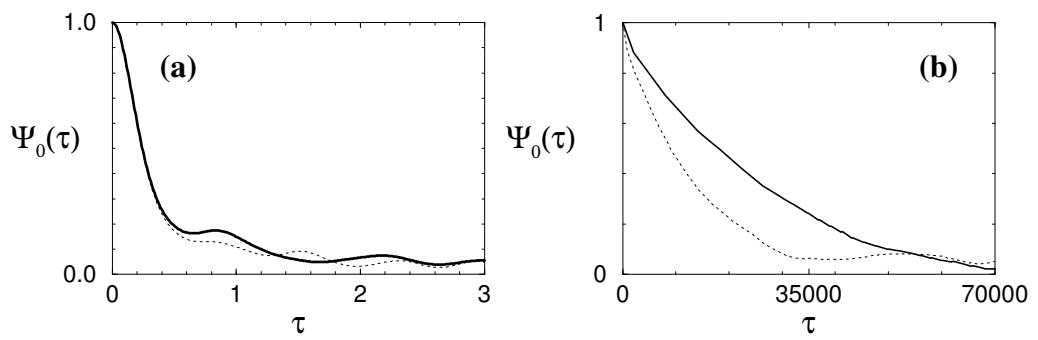

FIGURE 7. Envelopes of the normalized autocorrelation function $\Psi_{0}(\tau)$ for attractors in system (3). (a) $r=28$ and $D=0$ (solid line), and $D=0.01$ (dotted line); (b) $r=210, D=0$ (solid line), and $D=0.01$ (dotted line).

In the following, we summarize the main characteristics of the above mentioned classical models of random processes.

Harmonic noise is a stationary random process with zero mean. It is represented as follows $[78,79,80]$ :

$$
x(t)=R_{0}[1+\alpha(t)] \cos \left[\omega_{0} t+\phi(t)\right],
$$

where $R_{0}$ and $\omega_{0}$ are constant (average) values of the amplitude and frequency of oscillations, respectively; $\alpha(t)$ and $\phi(t)$ are random functions that characterize amplitude and phase fluctuations, respectively. The process $\alpha(t)$ is assumed to be stationary. Several simplifying assumptions that are most often used are as follows: (i) the amplitude and phase fluctuations are statistically independent, and (ii) the phase fluctuations $\phi(t)$ represent a Wiener process with a diffusion coefficient $B$. Under the assumptions made, the ACF of process (10) can be written as follows $[78,79,80]$ :

$$
\psi(\tau)=\frac{R_{0}^{2}}{2}\left[1+K_{\alpha}(\tau)\right] \exp (-B|\tau|) \cos \omega_{0} \tau
$$

where $K_{\alpha}(\tau)$ is the covariation function of reduced amplitude functions $\alpha(t){ }^{1}$. Using the Wiener-Khinchin theorem, one can derive the corresponding expressions for the spectral power density.

Generalized telegraph signal. This process describes random switchings between two possible states $x(t)= \pm a$. Two main kinds of telegraph signal are usually considered, namely, random and quasi-random telegraph signals [80, 81]. A random telegraph signal is characterized by a Poissonian distribution of switching moments $t_{k}$. The latter leads to the fact that the impulse duration $\theta$ has the exponential distribution:

$$
\rho(\theta)=n_{1} \exp \left(-n_{1} \theta\right), \theta \geq 0,
$$

where $n_{1}$ is the mean switching frequency. The ACF of such a process can be represented as follows:

$$
\psi(\tau)=a^{2} \exp \left(-2 n_{1}|\tau|\right) .
$$

Another type of telegraph signal (a quasi-random telegraph signal) corresponds to random switchings between the two states $x(t)= \pm a$, which can occur only in

\footnotetext{
${ }^{1}$ Prefactor $R_{0}^{2}\left[1+K_{\alpha}(\tau)\right]$ is the covariation function $K_{A}(\tau)$ of the random amplitude $A(t)=$ $R_{0}[1+\alpha(t)]$. This notion is most convenient to use in our future studies.
} 
discrete time moments $t_{n}=n \xi_{0}+\alpha, n=1,2,3, \ldots$, where $\xi_{0}=$ const and $\alpha$ is a random quantity. If the probability of switching events is equal to $1 / 2$, then the $\mathrm{ACF}$ of this process is given by the following expression:

$$
\begin{aligned}
& \psi(\tau)=a^{2}\left(1-\frac{|\tau|}{\xi_{0}}\right), \text { if }|\tau|<\xi_{0} \\
& \psi(\tau)=0, \text { if }|\tau| \geq \xi_{0} .
\end{aligned}
$$

5.1. Correlation and spectral analysis of spiral chaos. ¿From a physical viewpoint, chaotic attractors of the spiral type possess the properties of a noisy limit cycle. However, spiral attractors are realized in fully deterministic systems; that is, without external fluctuations. Consider the regime of spiral chaos in the Rössler system (2) for $a=b=0.2$ and $m=6.5$. Let us introduce the instantaneous amplitude $A(t)$ and phase $\Phi(t)$ according to the relations (7). We calculate the normalized autocorrelation function of the chaotic oscillations $x(t)$ (grey dots 1 ), the covariance function of the amplitude $K_{A}(t)$ and the effective phase diffusion coefficient $B_{\text {eff }}$. Figure 8 shows the results for $\Psi_{x}(\tau)$ in system (2) both without noise and in the presence of noise.The ACF decays almost exponentially both without noise (Fig. 8a) and in the presence of noise (Fig. 8b). Additionally, as seen from Fig. 8c, for $\tau<20$ there is an interval on which the correlations decrease much faster. Using Equation (11), we can approximate the envelope of the calculated ACF $\Psi_{x}(\tau)$. To do this, we substitute the numerically computed characteristics $K_{A}(\tau)$ and $B=B_{\text {eff }}$ into an expression for the normalized envelope $\Gamma(\tau)$ :

$$
\Gamma(\tau)=\frac{K_{A}(\tau)}{K_{A}(0)} \exp \left(-B_{\text {eff }}|\tau|\right)
$$
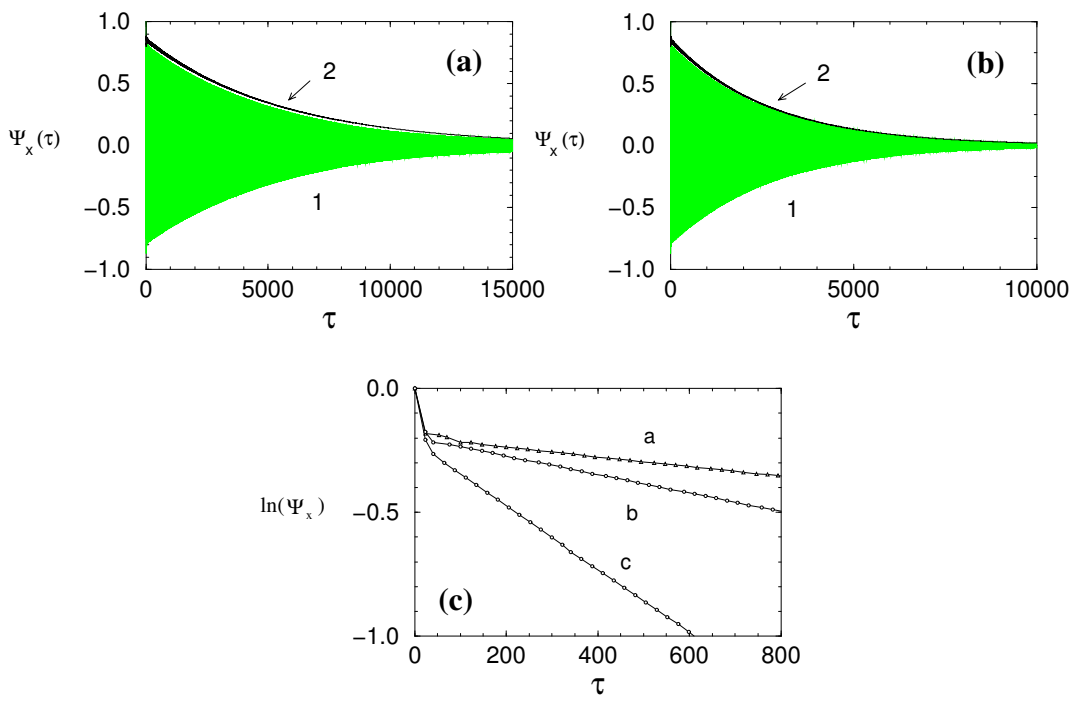

Figure 8. Normalized ACF of the $x(t)$ oscillations in system (2) for $m=6.5$ (dots 1) and its approximation by expression (15) (curves 2) for $D=0$ (a) and $D=10^{-3}$ (b). (c) The envelopes of ACF in a linear-logarithmic scale for $D=0$ (curve a), $D=0.001$ (curve b), and $D=0.01$ (curve c). 
The calculation results for $\Gamma(\tau)$ are shown in Figs. 8a and 8 b by curves 2. Equation (15) sufficiently describes the behavior of the envelope of $\Psi_{x}(\tau)$. Note that taking into account the multiplier $K_{A}(\tau) / K_{A}(0)$ enables us to obtain a good approximation for all times $\tau$. This means that the amplitude fluctuations play a significant role on short timeintervals, while the slow process of the correlation decay is mainlydetermined by the phase diffusion. Thus, we can observe a surprisingly good agreement between the numerical results for the spiral chaos and the data for the classical model of harmonic noise. At the same time, it is quite difficult to explain rigorously the reason for such a good agreement. Firstly, the relationship (11) was obtained by assuming the amplitude and phase values to be statistically independent. However, this approach cannot be applied to a chaotic regime. Secondly, when deriving (11), we used the fact that the phase fluctuations are described by a Wiener process. In the case of chaotic oscillations, $\Phi(t)$ is a more complicated process, and its statistical properties are unknown. It is especially important to note that the findings presented in Fig. 8a were obtained in the regime of purely deterministic chaos; that is, without noise in the system.

We have shown that for $\tau>\tau_{\text {cor }}$ the envelope of the ACF for the chaotic oscillations can be approximated by the exponential law $\exp \left(-B_{\text {eff }}|\tau|\right)$. Then according to the Wiener-Khichin theorem, the spectral peak at the average frequency $\omega_{0}$ must have a Lorenzian shape and its width is defined by the effective phase diffusion coefficient $B_{\text {eff }}$ :

$$
S(\omega)=C \frac{B_{\text {eff }}}{B_{\text {eff }}^{2}+\left(\omega-\omega_{0}\right)^{2}}, \quad C=\text { const. }
$$

The calculation results presented in Fig. 9 justify this statement. The basic spectral peak is approximated by using (16) and this fits quite well with the numerical results for the power spectrum of the $x(t)$ oscillations. We note that the findings shown in Figs. 8 and 9 for the noise intensity $D=10^{-3}$ have also been verified for different values of $D, 0<D<10^{-2}$, as well as for the range of parameter $m$ values that correspond to the regime of spiral chaos.

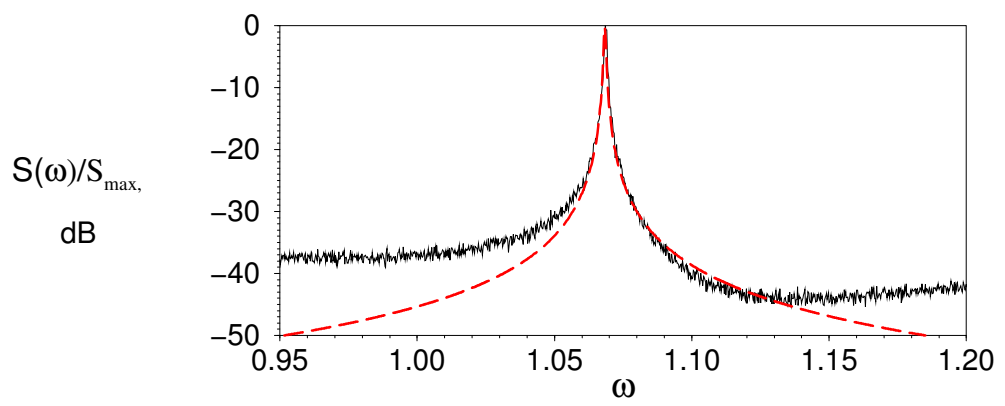

FiguRe 9. A part of the normalized power spectrum of $x(t)$ oscillations in system (2) for $a=b=0.2$, and $m=6.5$ (solid line) and its approximation by Equation (16) (dashed line) for the noise intensity $D=10^{-3}$.

Our findings for the approximation of the ACF and the shape of the basic spectral peak are completely confirmed by our investigations of spiral attractors in other dynamical systems [38, 39]. 
5.2. Correlation characteristics of the Lorenz attractor. In the previous section we have used the effective phase diffusion coefficient to describe the correlation properties of the Rössler system and the Anishchenko-Astakhov oscillator. However, such an approach cannot be applied to approximate autocorrelation functions of chaotic oscillations of a switching type. Some chaotic attractors demonstrating a rather complex structure can contain certain regions that are separated by manifolds of saddle points and cycles. Transitions (switchings) between these regions can occur provided that certain conditions are fulfilled [82]. Such oscillations can be observed, for example, in the Lorenz system [41]. Let us consider the Lorenz system in the regime of the quasi-hyperbolic attractor for $r=28, \sigma=10$, and $b=8 / 3$.

In the phase space of the Lorenz system there are two saddle-foci that are symmetrical about the $z$-axis and are separated by the stable manifold of a saddle point in the origin. This stable manifold has a complex structure that allows the trajectories to switch between the saddle-foci in specific paths [11, 82] (see Fig. 10). Unwinding about one of the saddle-foci, the trajectory approaches the stable manifold and then can jump to the other saddle-focus with a certain probability. The rotation about the saddle-foci does not contribute considerably to the decay of the $\mathrm{ACF}$, while the frequency of "random" switchings essentially affects the rate of the ACF decay. Consider the time series of the $x$ coordinate of the Lorenz system, that is shown in Fig. 11. If one introduces a symbolic dynamics; that is, one excludes the rotation about the saddle-foci, one obtains a telegraph-like signal. Figure 12 shows the ACF of the $x(t)$ oscillations for the Lorenz attractor and the ACF of the corresponding telegraph signal. Comparing these two figures, we can state that the time of the correlation decay and the behavior of the ACF on this time scale are predominantly determined by switchings, whereas the rotation about the saddlefoci makes a minor contribution to the $\mathrm{ACF}$ decay on large times. It is worth noting that the ACF decreases linearly on short times. This fact is remarkable. because the linear decay of the ACF corresponds to a discrete equidistant residence time probability distribution in the form of delta-peaks. Additionally, the probability of switchings between the two states is equal to $1 / 2[80,81]$.

Figure 13 shows the residence time distribution calculated for the telegraph signal resulting from switchings in the Lorenz system. As Fig. 13a shows, the residence

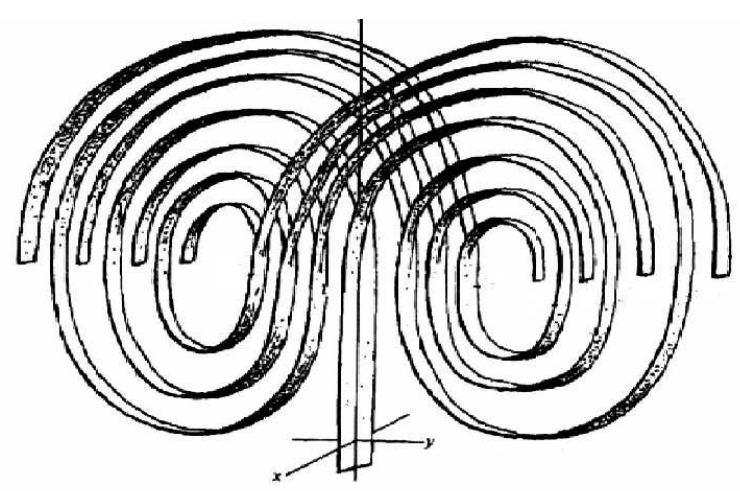

Figure 10. Qualitative illustration of the structure of manifolds in the Lorenz system 


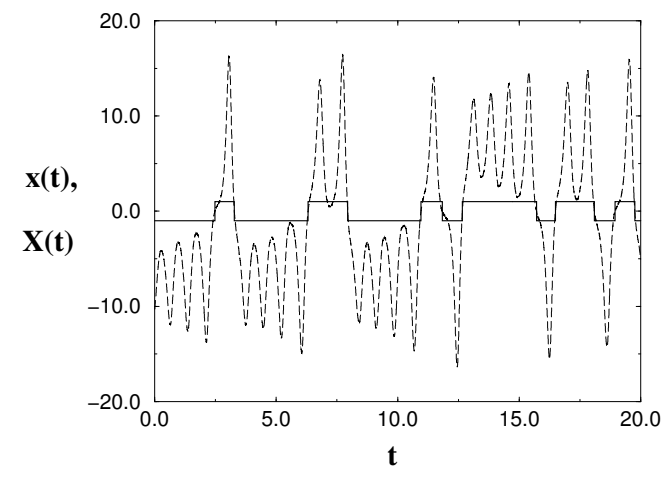

FIGURE 11. Telegraph signal (solid curve) obtained for the $x(t)$ oscillations (dashed curve) of the Lorenz system at $\sigma=10, \beta=$ $8 / 3$, and $r=28$.
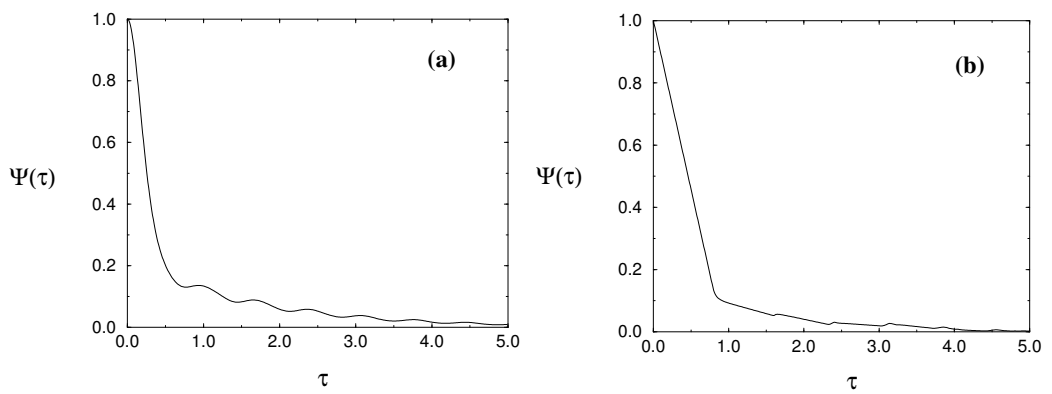

Figure 12. The ACF of the $x(t)$ oscillations (a) and of the telegraph signal (b).

time distribution in the two attractor regions has a structure that is quite similar to an equidistant discrete distribution. At the same time the peaks are characterized by a finite width. Figure 13b represents the probability distribution of switchings that occur at multiples of $\xi_{0}$, where $\xi_{0}$ is the minimal residence time in one of the states. This dependence shows that the probability of transition at time $\xi_{0}$ is close to $1 / 2$. The discrete character of switchings can be explained by peculiarities of the structure of the manifolds of the Lorenz system (see Fig. 10). In the vicinity of the origin $x=0, y=0$, the manifolds split into two leaves. This leads to the fact that the probability of switchings between the two states in one revolution about the fixed point is approximately equal to $1 / 2$. This particular aspect of the dynamics ensures that the ACF of the $x(t)$ and $y(t)$ oscillations on the Lorenz attractor has the form defined by expression (14). However, the finite width of the peaks in the distribution and deviations from the probability $1 / 2$ can lead to an ACF that decays to a certain finite, nonvanishing value.

6. Autocorrelation function and power spectrum of spiral chaos in a physical experiment. The experiments were conducted on an experimental unit that consisted of a radio-technical generator with inertial nonlinearity (the Anishchenko-Astakhov oscillator) [43] having the basic frequency $18.5 \mathrm{kHz}$, a computer with a fast analog-digital convertor (ADC) with the discretization frequency 

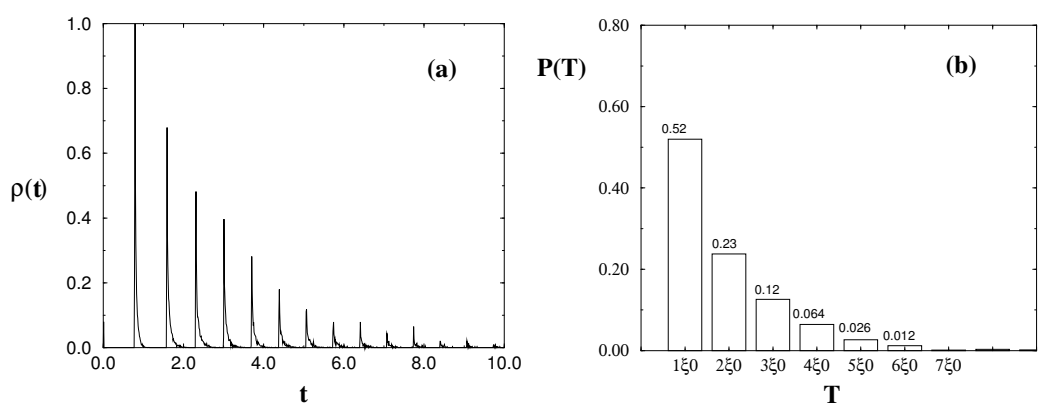

FIGURE 13. The distribution of impulse durations of the telegraph signal (a) and probabilities of transitions at times multiple to $\xi_{0}$ (b).

$694.5 \mathrm{kHz}$, and a Gaussian broadband noise generator with a frequency range from $0 \mathrm{kHz}$ to $100 \mathrm{kHz}$ [83]. The behavior of the ACF was also analyzed in the presence of noise. With this purpose, a broadband noise from the external noise generator was applied to the system, and the noise intensity can be varied. The oscillator with inertial nonlinearity is described by a simple three-dimensional dynamical system:

$$
\dot{x}=m x+y-x z-\delta x^{3}, \dot{y}=-x, \dot{z}=-g z+g I(x) x^{2}, I(x)= \begin{cases}1, & x>0 \\ 0, & x \leq 0 .\end{cases}
$$

The system (17) can demonstrate the regimes of spiral chaos for certain values of parameters $m$ and $g$ [43].

The first important question to be uniquely answered by the experiment is whether a Wiener process approach can be applied to describe the phase of the $x(t)$ process, as assumed in [21, 37, 39, 75]. To define the diffusion coefficient $B_{\text {eff }}$, the instantaneous phase is introduced by using an analytical signal concept and performing the Hilbert transform for experimental realizations $x(t)$ [77]. Then the phase variance $\sigma_{\Phi}^{2}(t)$ is calculated by averaging over an ensemble of $N$ realizations. The effective phase diffusion coefficient is defined by the rate of the variance growth in time. The temporal dependence of the phase diffusion shown in Fig. 14 is not rigorously linear as it must be observed for the Wiener process. However, the linear growth dominates over small-scaled oscillations of the phase variance. Thus, the process under consideration can be related to a Wiener process with diffusion coefficient $B_{\text {eff }}$. The linear dependence defining the effective diffusion coefficient is found by the least-square method.

The next step in our experiment is to measure the ACF of chaotic oscillations of the oscillator with inertial nonlinearity. Several tens of the signal $x(t)$ realizations, each of $10 \mathrm{sec}$ duration, were registered by the fast ADC. The total length of realization is $3 \div 5 \times 10^{5}$ oscillation periods with the discretization step $\Delta t$ corresponding to 37 points per period. The ACF is calculated as follows. First, we compute the time-average value of the $x$ variable for each of the $N$ realizations of the $x(t)$ process:

$$
\bar{x}=\frac{1}{n} \sum_{i=1}^{n} x\left(t_{i}\right)
$$




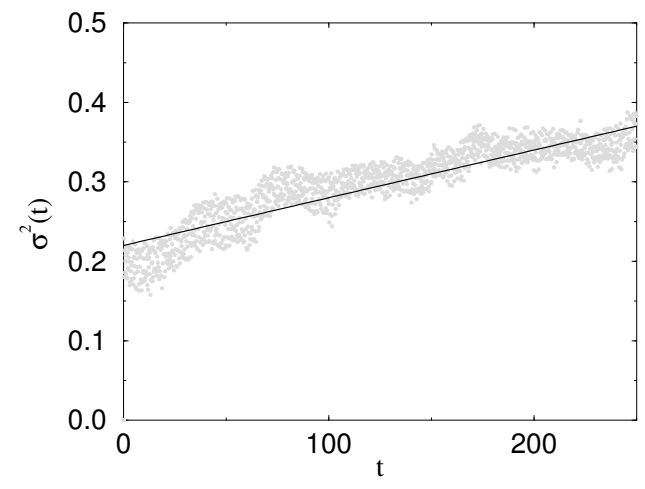

FiguRE 14. Temporal dependence of the phase variance in the presence of noise with a root-mean square value of the noise intensity $D=0.001 \mathrm{mV}$ and its linear approximation by the least-square method (time $t$ is a dimensionless variable and equals to the number of periods of oscillations).

Then, we find the mean product $\langle(x(t) x(t+\tau))\rangle$ by averaging over time:

$$
K_{l}(\tau)=\frac{1}{p} \sum_{i=1}^{p} x\left(t_{i}\right) x\left(t_{i}+k \Delta t\right), \tau=k \Delta t_{i}, k=0,1, \ldots, n-p,
$$

where $l=1, \ldots, N$ is the number of realization. When calculating one may encounter a problem that is connected to the limitation of a number of $x\left(t_{i}\right)$ values, $i=1,2, \ldots, n$, that are stored in the ADC buffer. The time-averaging result is converged if the number of averagings $p$ is sufficiently large. On the other hand, the larger the chosen $p$, the smaller the time $\tau_{\max }=(n-p) \Delta t$ for the ACF estimation. Becuase the rate of correlation splitting is not high in the regime being considered, the ACF must be computed on a very large time interval. For this reason, the value of $p$ was chosen to be not too large. To attain a high precision of the ACF calculation the obtained data were further averaged over $N$ realizations:

$$
\psi(\tau)=\frac{1}{N} \sum_{l=1}^{N} K_{l}(\tau)-\bar{x}^{2} .
$$

The ACF was normalized on its maximal value at $\tau=0$; that is, $\Psi(\tau)=\psi(\tau) / \psi(0)$. Figure 15 illustrates logarithmic plots of normalized ACF envelopes that were found experimentally for different values of the external noise intensity. The obtained dependences were approximated according to the exponential law $\Psi_{\text {app }}(\tau)=$ $\exp \left(-B_{\text {eff }} \tau\right)$, where $B_{\text {eff }}$ is the experimentally found effective diffusion coefficient of the instantaneous phase. The approximation plots are shown in Fig. 15 by symbols.

Now let us analyze the results of the power spectrum measurements. The power spectrum of a diffusive process looks like a Lorenzian having the width that is defined by the effective phase diffusion coefficient. For the normalized spectrum the Lorenzian is given by expression (16). In experiment, the effective diffusion coefficient can be independently defined by measuring the spectral peak width. To obtain a more precise value of the diffusion coefficient, we approximate the spectral 


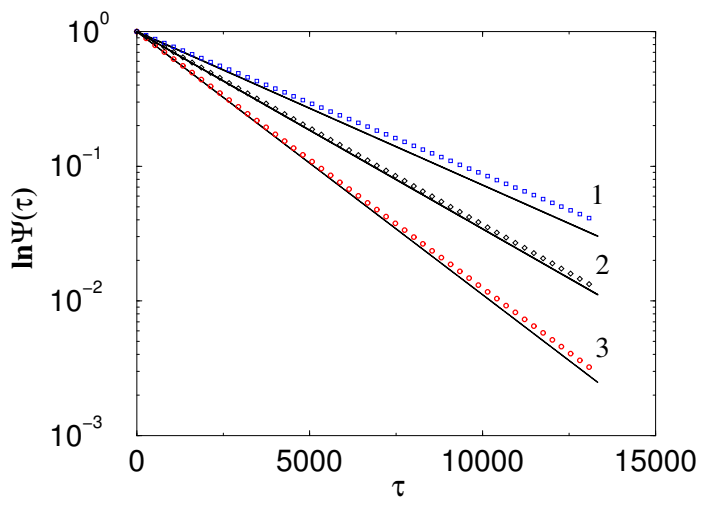

Figure 15. ACF envelopes (solid lines) obtained experimentally for different root-mean-square values of the external noise intensity: $1-D=0,2-D=0.0005 \mathrm{mV}$, and $3-D=0.001 \mathrm{mV}$; and their exponential approximations (dashed lines) with the decrement of decay $B_{\text {eff }}=0.00024, B_{\text {eff }}=0.00033$, and $B_{\text {eff }}=0.000439$, respectively. The other parameters for numeric calculations are $N=100$, $n=262144$, and $p=1 / 2 n$.

peak with the formula (16) by varying $B_{\text {eff }}$. The resulting value of the coefficient will be the one at which the approximation error is minimal (see Fig. 16a).

Figures 16a and 16b illustrates parts of the experimental power spectra of the GIN without and in the presence of external noise sources. The spectrum was calculated by means of a standart FFT method with averaging. The window length was about $2^{18}$ points, and the total number of windows was about 50 . The main result is that the effective phase diffusion coefficient values estimated from the spectra correspond with the values obtained from the linear approximation of the growth of the instantaneous phase variance. The corresponding phase diffusion coefficient values are given in Table 1 for three different levels of the external noise.
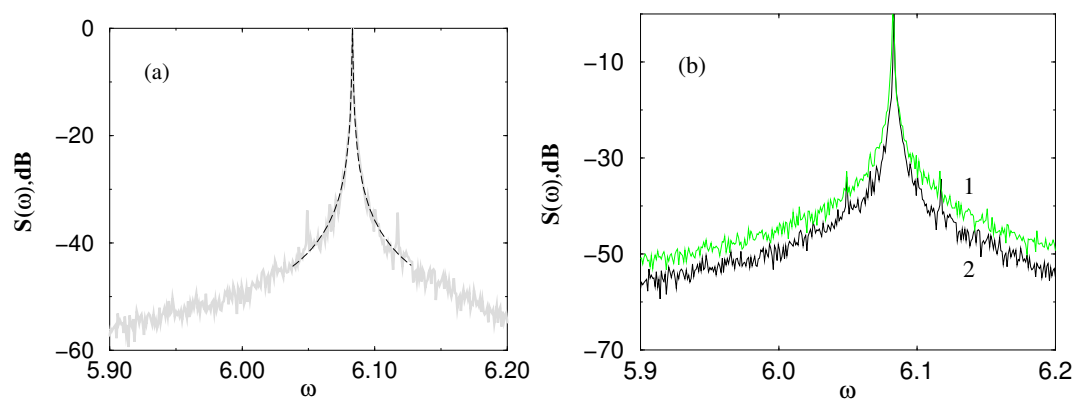

FiguRE 16. (a) Experimental power spectrum of the $x(t)$ oscillations in system (17) and its theoretical approximation by (16) with $B_{\text {eff }}=0.00033$ in the presence of noise with $D=0.0005$; and (b) Power spectra for $D=0.001$ (curve 1 ) and $D=0$ (curve 2). 


\begin{tabular}{|r|r|r|}
\hline$D, \mathrm{mV}$ & $B_{\text {eff }}$ (Hilbert) & $B_{\text {eff }}$ (Spectrum) \\
\hline 0 & 0.000244 & 0.000266 \\
0.0005 & 0.00033 & 0.000342 \\
0.001 & 0.000439 & 0.000443 \\
\hline
\end{tabular}

TABle 1. Comparison of phase diffusion coefficient values obtained by different methods without and in the presence of noise with different intensities.

Thus, it has been experimentally established that in the regime of spiral chaos the instantaneous phase variance of chaotic oscillations grows, on average, linearly with the diffusion coefficient $B_{\text {eff }}$. Without noise this coefficient is defined by the chaotic dynamics of the system. In the presence of noise the growth of the phase variance is also linear but the $B_{\text {eff }}$ value increases. The ACF of the spiral chaos decays in time according to the exponential law $\exp \left(-B_{\text {eff }} \tau\right)$. The spectral linewidth of oscillations at the basic frequency $\omega_{0}$ is defined by the effective phase diffusion coefficient from expression (16).

7. Conclusion. In our studies, we have shown that there is a group of nonhyperbolic attractors of the spiral type for which noise strongly influences the characteristics of the relaxation to a stationary distribution as well as the correlation time and barely changes the positive Lyapunov exponent.

The rate of mixing on nonhyperbolic attractors in $\mathbf{R}^{3}$ is determined not only by the positive Lyapunov exponent but also by the instantaneous phase dynamics of chaotic oscillations. In the regime of spiral chaos noise causing phase changes can essentially accelerate the relaxation to a stationary distribution.

For chaotic attractors with a nonregular behavior of the instantaneous phase, the rate of mixing cannot be considerably affected by noise. This statement is true for nonhyperbolic attractors of the funnel type and for the attractors of the switching type; for example, for the quasi-hyperbolic Lorenz attractor.

We have shown numerically and experimentally that the spiral chaos retains to a great extent the spectral and correlation properties of quasi-harmonic oscillations. With this, the rate of correlation splitting in a differential system depends on short times on the instantaneous amplitude and the instantaneous phase diffusion. The width of the basic peak in the power spectrum of the spiral chaos is correspondingly defined by $B_{\text {eff }}$ and oscillations of the instantaneous amplitude determine the level of the spectrum background. The effective phase diffusion coefficient in a noise-free system is defined by its chaotic dynamics but is not directly related to the positive Lyapunov exponent. Our studies of the statistical properties of the Lorenz attractor have demonstrated that the properties of the ACF are mainly defined by a random switching process and slightly depend on the rotation about the saddle-foci. The classical model of telegraph signal enables one to describe the behavior of $\psi(\tau)$ for the Lorenz attractor by using expression (14). In particular, this expression closely approximates a linear decay of the ACF from 1.0 to 0.2 that allows us to estimate theoretically the correlation time. The power spectrum of the Lorenz attractor in a flow and in the Poincaré map was studied in [36] by applying the symbolic dynamics methods. We have already established in this study that the 
power spectrum is not a Lorenzian. Our results, obtained by using the model of telegraph signal are consistent with the findings presented in [36].

Acknowledgments. This work was partially supported by the U.S. Civilian Research and Development Foundation (Award No. SR-006-X1) and the Russian Ministry of Education (grant no. E02-3.2-345), INTAS No. 01-2061 (V.S. Anishchenko and T.E. Vadivasova), INTAS YSF 2002-3 Renewal and the RF President grant MK-2833.2004.2 (G.I. Strelkova).

\section{REFERENCES}

[1] D.V. Anosov, Geodesic flows on Closed Riemannian manifolds with a negative CuRvature. Proc. Steklov Math. Inst. 90(1967) 3-209 (in Russian).

[2] S. Smale, Differentiable Dynamical Systems. Bull Amer Math Soc 73(1967) 747-817.

[3] D. Ruelle and F. Takens, On the nature of turbulence. Commun Math Phys 20(1971) 167-92.

[4] J. Guckenheimer and P. Holms, Nonlinear Oscillations,Dynamical Systems, and BifurCAtions of Vector Fields. Springer-Verlag, New York, 1983.

[5] Ya.G. Sinai, Dynamical systems with elastic collisions. Russian Math Surveys 25(1970) 141-92.

[6] Ya.G. Sinai, Stochasticity of Dynamical Systems. In Nonlinear Waves, edited by A.V. Gaponov-Grekhov, Nauka, Moscow, 1979. P. 192 (in Russian).

[7] L.A. Bunimovich and Ya.G. Sinai. In Nonlinear Waves, edited by A.V. Gaponov-Grekhov, Nauka, Moscow, 1980. P. 212 (in Russian).

[8] J.-P. Eckmann, D. Ruelle, Ergodic theory of Chaos And Strange ATtractors. Rev Mod Phys 57(1985) 617.

[9] D. Ruelle, A measure associated with Axiom A attractors. Am J Math 98(1976) 619.

[10] D. Ruelle, Bol Soc Bras Math 9(1978) 83.

[11] L.P. Shilnikov, Mathematical problems of nonlinear dynamics: a tutorial. Int J of Bif and Chaos 7(1997) 1953; L.P. Shilnikov, Strange ATTraCtors AND DyNAmical Models. J of Circuits, Systems, and Computers 3(1993) 1-10.

[12] V.S. Afraimovich and L.P. Shilnikov, Strange atTractors And Quasiattractors. In Nonlinear Dynamics and Turbulence, ed. by G.I. Barenblatt, G. Iooss, and D.D. Joseph. Pitman, Boston, London, Melbourne, 1983. P. 1-34.

[13] V.S. Anishchenko and G.I. Strelkova, Irregular attractors. Discrete Dyn Nat Soc 2(1998) 53-72.

[14] R. Graham, W. Ebeling (private communications); R. Graham, A. Hamm, and T. Tel, Nonequilibrium POTENTIALS FOR DYNAMICAL SYSTEMS WITH FRACTAL ATTRACTORS OR REPELLERS. Phys Rev Lett 66(1991) 3089.

[15] E. Ott, E.D. Yorke, and J.A. Yorke, A Scaling Law: How an Attractor's Volume DePENDS ON NoISE LEVEL. Physica 16D(1985) 62.

[16] Ch.G. Schroer, E. Ott, and J.A. Yorke, Effect of nOISE on nonhyperbolic ChaOtic ATTRACTORs. Phys Rev Lett 81(1989) 1397.

[17] T. Sauer, C. Grebogi, and J.A. Yorke, How LONG Do NumeriCAl CHAOtiC SOlutions REMAin VALID? Phys Rev Lett 79(1997) 59-62.

[18] L. Jaeger and H. Kantz, Homoclinic tangencies And nonnormal Jacobians - EFFECTs of NOISE IN NONHYPERBOLIC CHAOTIC SYSTEMS. Physica 105D(1997) 79.

[19] Yu. Kifer, Commun Math Phys 121(1989) 445. Yu.I. Kifer, Izv Akad Nauk USSR. Ser Math 38(1974) 1091.

[20] S.M. Hammel, J.A. Yorke, and C. Grebogi, Do NumericAl ORBITs of ChaOtiC DYNAMiCAL PRocesses REPRESENT TRUe orbits? J of Complexity 3(1987) 136-45 ; S.M. Hammel, J.A. Yorke, and C. Grebogi, Numerical orbits of Chaotic processes Represent true orbits. Bull Am Math Soc 19(1988) 465-69.

[21] V.S. Anishchenko, T.E. Vadivasova, A.S. Kopeikin, J. Kurths, and G.I. Strelkova, EFFECT OF NOISE ON THE RELAXATION TO AN INVARIANT PROBABILITY MEASURE OF NONHYPERBOLIC CHaOtic ATtractors. Phys Rev Lett 87(2001) 054101. 
[22] V.S. Anishchenko, T.E. Vadivasova, A.S. Kopeikin,J. Kurths, and G.I. Strelkova, PECULIARITIES OF THE RELAXATION TO AN INVARIANT PROBABILITY MEASURE OF NONHYPERBOLIC ATtractors in the Presence of nOISE. Phys Rev E 65(2002) 036206.

[23] G.M. Zaslavsky, Chaos in Dynamical Systems. Harwood Acad. Publishers, New York, 1985.

[24] P. Billingsley, Ergodic Theory and Information. John Wiley and Sons, Inc., New York, 1965.

[25] I.P. Cornfeld, S.V. Fomin, Ya. Sinai, Ergodic Theory. Springer-Verlag, New York, 1982.

[26] A.N. Kolmogorov, About the entropy per time unit as an metric invariant of autoMORPhisms. Dokl Acad Nauk USSR 124(1959) 754-55 (in Russian).

[27] Ya. Sinai, About the notion of entropy of Dynamical systems. Dokl Acad Nauk USSR 124(1959) 768-71 (in Russian).

[28] Ya. Pesin, Lyapunov Characteristics exponents And smooth ERGodic theOry. Russian Math Surveys 32(1977) 55.

[29] R. Bowen, Equilibrium States and the Ergodic theory of Anosov Diffeomorphisms. Lect. Notes in Mathematics. Springer-Verlag, Berlin, Heidelberg, 1975.

[30] M.L. Blank, Stability and Localization in Chaotic Dynamics. Moscow Center for Cont. Math. Educ., Moscow, 2001 (in Russian).

[31] D. Ruelle, The thermodynamic formalism for Expanding MAPs. Math Phys 125(1989) 239.

[32] F. Christiansen, G. Paladin, H.H. Rugh, Determination of Correlation spectra in CHaOtic Systems. Phys Rev Lett 65(1990) 2087.

[33] C. Liverani, Decay of Correlations. Ann Math 142(1995) 239.

[34] G. Froyland, Computer-Assisted Bounds for the RAte of DeCAY of Correlations. Comm Math Phys 189(1997) 237.

[35] R. Bowen, D. Ruelle, The ergodic theory of Axiom A flows. Inventions Math. 29(1975) 181.

[36] R. Badii, M. Finardi, G. Broggi, M.A. Sepúlveda, Physica D58(1992) 304.

[37] V.S. Anishchenko, T.E. Vadivasova, G.A. Okrokvertskhov, G.I. Strelkova, Correlation ANALYSis OF DYNAMiCAl CHAOS. Physica A325(2003) 199-212.

[38] V.S. Anishchenko, T.E. Vadivasova, A.S. Kopeikin, J. Kurths, G.I. Strelkova, Spectral AND Correlation Analysis of Spiral Chaos. Fluct Noise Lett 3(2003) L213-21.

[39] V.S. Anishchenko, T.E. Vadivasova, G.A. Okrokvertskhov, G.I. Strelkova, Correlation ANALYSis OF THE REGIMES OF DETERMinistic AND NOISY CHAOS. J Comm Techn Electr 48(2003) 750-60.

[40] O.E. Rössler, An equation for continuous chaos. Phys Lett A57(1976) 397-98.

[41] E.N. Lorenz, Deterministic non-Periodic flow. J Atmos Sci 20(1963) 130-41.

[42] V.S. Anishchenko, Complex Oscillations in Simple Systems. Nauka, Moscow, 1990 (in Russian).

[43] V.S. Anishchenko, Dynamical Chaos - Models and Experiments. World Scientific, Singapore, 1995

[44] R.F. Williams, In: Proceedings of the Symposium in Pure Mathematics: Global AnalYSIS. Am. Math. Soc., 1970. Vol. 14, p. 361.

[45] R. Plykin, On hyperbolic ATtractors of Diffeomorphisms. Mathematics - Uspekhi 35(1980), 94-104 (in Russian).

[46] V.S. Afraimovich, V.V. Bykov, and L.P. Shilnikov, On Appearance And Structure of Lorenz ATtractor. Dokl Acad Nauk USSR 234(1977) 336 (in Russian).

[47] L.P. Shilnikov, In: Methods of Qual. Theory of Differential Equations. Gorky State University, Gorky, 1989. P. 130 (in Russian).

[48] R. Lozi, Un Attracteur Etrange du Type Attracteur de Henon. J de Physique 39(1978) 9-10.

[49] V.N. Belykh, Chaotic and strange attractors of a two-Dimensional map. Math USSR Sb 186(1995) 311-18 (in Russian).

[50] S. Banerjee, J. Yorke, and C. Grebogi, Robust Chaos. Phys Rev Lett 80(1998) 3049-52.

[51] V.S. Anishchenko, T.E. Vadivasova, G.I. Strelkova, and A.S. Kopeikin, ChнотiC AtтraCtors of Two-dimensional Invertible Maps. Discrete Dynamics in Nat and Soc 2(1998) 249-54.

[52] Y.-C. Lai, C. Grebogi, and J. Kurths, Modeling of Deterministic Chaotic Systems. Phys Rev E 59(1999) 2907-10.

[53] Y.-C. Lai and C. Grebogi, Modeling of Coupled chaotic oscillators. Phys Rev Lett 82(1999) 4803-06. 
[54] E. Ott, Chaos in Dynamical Systems. Cambridge University Press, 1993.

[55] S.P. Dawson, C. Grebogi, T. Sauer, and J. A. Yorke, Obstructions to Shadowing When A Lyapunov exponent fluctuates about zero. Phys Rev Lett 73(1994) 1927-930.

[56] S.P. Dawson, Strange Nonattracting Chaotic Sets, Crises, and Fluctuating LyaPunov Exponents. Phys Rev Lett 76(1996) 4348-351; P. Moresco and S.P. Dawson, ChaOs AND CRISES In MORE THAN TWO Dimensions. Phys Rev E 55(1997) 5350-360.

[57] E.J. Kostelich, I. Kan, C. Grebogi, E. Ott, and J. A. Yorke, Unstable dimension variabilITY: A SOURCE OF NONHYPERBoliCity In CHAOTIC SYSTEMS. Physica D109(1997) 81-90.

[58] Y.-C. Lai, C. Grebogi, J.A. Yorke, and I. Kan, How often ARE ChaOtiC SAdDles nonhyPERBOLIC? Nonlinearity 6(1993) 779-97.

[59] V.S. Anishchenko, A.S. Kopeikin, J. Kurths, T.E. Vadivasova, G.I. Strelkova, StudYING Hyperbolicity of Chaotic Systems. Phys Lett A 270(2000) 301-07.

[60] Chua's circuit: A paradigm for chaos, ed. by R.N. Madan. World Scientific, Singapore, 1993.

[61] N.K. Gavrilov and L.P. Shilnikov, On three-Dimensional DynamiCAl Systems Being Close to A NON-ROBUSt homocLINIC CURVE. Math USSR Sb 88(1972) 475-92; 90(1973) 139-56 (in Russian).

[62] R.L. Stratonovich, In: Noise in Nonlinear Dynamical Systems. Vol. 1. Cambridge University Press, 1989. P. 16

[63] W. Horsthemke and R. Lefever, Noise-induced transitions. Springer-Verlag, Berlin, Heidelberg, 1984.

[64] C.V. Gardiner, Handbook of Stochastic Methods for Physics, Chemistry, and the Natural ScIEnces. Springer-Verlag, Berlin, Heidelberg, 1983.

[65] H. Haken, Advanced Synergetics. Springer-Verlag, Berlin, Heidelberg, 1983.

[66] R. Graham, In: Noise in Nonlinear Dynamical Systems. Vol. 1. Theory of Continuous Fokker-Planck Systems, eds. F. Moss, P.V.E. McClintock. Cambridge University Press, Cambridge, 1988.

[67] V.S. Anishchenko, W. Ebeling, Effects of Strong NOISE on ATtractors of DyNAMiCAL Systems. Z Phys B 81(1990) 445-50.

[68] J. Guckenheimer, Noise in Chaotic systems. Nature 298(1982) 358-61.

[69] C.S. Hsu and M.C. Kim, J Stat Phys 38(1985) 735.

[70] Y.G. Sinai, Finite-dimensional Randomness. Russian Math Surveys 46(1991) 147-59.

[71] V.S. Anishchenko and A.B. Neiman, Noise-Induced transition in the Lorenz Model. Sov Tech Phys Lett 17(1991) 43-47.

[72] V.S. Anishchenko and A.B. Neiman, Structure And properties of Chaos in the PRESEnCE of noise. In: NonlinearDynamics of Structures, edited by R.Z. Sagdeev, et al. World Scientific, Singapore, 1991. P. 21.

[73] D. Alonso, D. MacKernon, P. Gaspard, and G. Nicolis, Statistical approach to nonhyPERBOlic ChaOtic SYSTEMs. Phys Rev E 54(1996) 2474-78.

[74] V.S. Anishchenko et al., Nonlinear Dynamics of Chaotic and Stochastic Systems. Springer-Verlag, Berlin, Heidelberg, 2002.

[75] J.D. Farmer, Spectral Broadening of Period-Doubling Bifurcation Sequences. Phys Rev Lett 47(1981) 179-82.

[76] A. Arneodo, P. Collet, and C. Tresser, Possible new strange attractors with spiral Structure. Commun Math Phys 79(1981) 573.

[77] M. Rosenblum, A. Pikovsky, J. Kurths, Phase synchronization of Chaotic oscillations. Phys Rev Lett 76(1996) 1804-807.

[78] R.L. Stratonovich, Topics of Random Noise. Gordon and Breach, New York, Vol. 1 1963, Vol. 21967.

[79] A.N. Malakhov, Fluctuations in Autooscillating Systems. Nauka, Moscow 1968 (in Russian).

[80] S.M. Rytov, Introduction in Statistical Radiophysics. Nauka, Moscow 1966 (in Russian).

[81] V.I. Tikhonov, M.A. Mironov, Markovian Processes. Sov. Radio, Moscow 1977 (in Russian).

[82] E.A. Jackson, Perspectives of Nonlinear Dynamics. Cambridge University Press, Vol. 1 1989, Vol. 21991.

[83] V.S. Anishchenko, T.E. Vadivasova, J. Kurths, G.A. Okrokvertskhov, G.I. Strelkova, AutoCORRELATION FUNCTION AND SPECTRAL LINEWIDTH OF SPIRAL CHAOS IN A PHYSICAL EXPERIMENT. Phys Rev E 69(2004) (in print). 
184 V.S. ANISHCHENKO, T.E. VADIVASOVA, G.I. STRELKOVA, G.A. OKROKVERTSKHOV

Received on Jan. 30, 2004. Revised on March 23, 2004.

E-mail address: wadim@chaos.ssu.runnet.ru

E-mail address: tanya@chaos.ssu.runnet.ru

E-mail address: galya@chaos.ssu.runnet.ru

E-mail address: george@chaos.ssu.runnet.ru 